\title{
Clima organizacional, ¿afecta al estrés laboral?: comparación entre funcionarios de la salud y seguridad pública
}

\section{Organizational climate, does it affect work stress? comparison between public health and safety officials}

\author{
María Margarita Chiang Vega ${ }^{*_{1}}$ \\ Universidad del Bío-Bío \\ mchiang@ubiobio.cl \\ Camila Sanhueza Hernández ${ }^{1}$ \\ Universidad del Bío-Bío \\ cafsanhu@egresados.ubiobio.cl \\ María José Rivera Cerda ${ }^{1}$ \\ Universidad del Bío-Bío \\ mirivera@egresados.ubiobio.cl
}

* Autor Corresponsal

1 Universidad del Bío-Bío, Facultad de Ciencias Empresariales, Departamento de Administración y Auditoría, Avenida Collao 1202 Casilla 5-C, Concepción, Región del Biobio. CHILE.

\footnotetext{
Resumen

Propósito: la presente investigación busca conocer el efecto de los factores del clima organizacional en el estrés laboral, comparando funcionarios públicos del área de la salud y seguridad.

Diseño/metodología: la muestra corresponde a 321 funcionarios de instituciones públicas. Se han estudiado dos casos: el primero considera a funcionarios de salud $(n=186)$ y el segundo considera a funcionarios de seguridad pública $(n=135)$, para analizar si existen diferencias entre ambas áreas de trabajo. El estudio es de tipo transversal, descriptivo y correlacional. Los datos se obtuvieron por medio de la aplicación de una encuesta que integra dos cuestionarios: el de clima organizacional (Koys y Decottis, 1991, adaptado y validado por Chiang, Salazar, Huerta y Núñez, 2008) y el cuestionario de estrés laboral (Karasek, 1979).

Resultados: se cumple la hipótesis planteada en este estudio: el estrés laboral sí es afectado por el clima organizacional, pero en diferentes grados dependiendo de la dimensión y tipo de funcionario.

Implicaciones: la dimensión apoyo del clima organizacional afecta al estrés principalmente en el área de salud y la dimensión innovación afecta más a los funcionarios de seguridad pública.

Originalidad/valor: las variables en estudio pueden interferir en el desarrollo de las labores de los empleados, por lo que es relevante su análisis para lograr una buena gestión de los recursos humanos, con el fin de cumplir con los objetivos y metas de la organización.
}

INFORMACIÓN ARTÍCULO

Recibido: 23 de Octubre 2021

Aceptado: 20 de Diciembre 2021
Palabras Claves:

Clima organizacional

Estrés laboral

Calidad de vida laboral

Servicios públicos

Funcionarios públicos 


\begin{abstract}
Purpose: this research seeks to know the effect of organizational climate on work stress by comparing health care and safety public officials.

Design/methodology: the sample corresponds to 321 officials of public institutions, two cases were studied: the first considers health care public officials $(n=186)$ and the second security public officials ( $n=135)$, to analyze if there are differences between the two work areas. The study is crosssectional, descriptive and correlational. The data were obtained through the application of a survey that integrates two questionnaires: organizational climate (Koys and Decottis, 1991, adapted and validated by Chiang, Salazar, Huerta and Núñez, 2008) and questionnaire of work stress (Karasek, 1979). Results: the hypothesis proposed in this study is fulfilled, since work stress is affected by the organizational climate, but at different degrees depending on the factors and type of employee.

Implications: the support dimension of the organizational climate affects stress mainly in the health care and the innovation dimension affects more public security officials.

Originality/value: the variables under study can interfere in the development of the employees' work, reason why their analysis is relevant to achieve a good management of human resources, in order to meet the objectives and goals of the organization.
\end{abstract}

\section{ARTICLE INFO}

Received: 23 October 2021

Accepted: 20 December 2021

\section{INTRODUCCIÓN}

Es una necesidad de la organización identificar aquellos factores que originan las deficiencias existentes en instituciones públicas. Por ello, Aguirre (1990) señala que evaluar las percepciones tanto de los usuarios como del trabajador, representa una oportunidad de mejora en la gestión de servicios públicos.

En tanto, la salud es considerada un factor insustituible que influye en el bienestar social, el desarrollo, la economía y la educación de los pueblos (Malagón, Galán y Pontón, 2008), y estos aspectos obligan a las instituciones hospitalarias a ofrecer atención médica oportuna, aceptable, asequible y de calidad satisfactoria (Organización Mundial de la Salud, 2013).

En estos tiempos cambiantes donde se ha evolucionado en función del momento histórico, de las culturas, del sistema social y del nivel de conocimientos, el sistema de salud se ha convertido en una de las áreas más importantes de la actividad humana. Por ejemplo, en Chile el aumento en la esperanza de vida y las enfermedades crónicas con una alta prevalencia ha ocasionado una sobrecarga en el sistema de salud privado como estatal (Ganga, Piñones, González y Rebagliati, 2016).

En este sentido, Rosas (2012, citado en Montero, 2015) plantea que la seguridad pública es el mantenimiento de la paz, la ley y el orden público.
Por otra parte, Yáñez (2003) señala que la fuerza pública no solo está conformada por los funcionarios de seguridad, sino que también se compone de la aplicación de sanciones administrativas, penales y ejecución de resoluciones judiciales. Por tanto, es importante conocer las perspectivas de la salud y de la seguridad pública, debido a que es motivo de preocupación en los gobiernos de todos los países. En concordancia, Arnoletto y Díaz (2009) expresan que los organismos públicos cargan sobre su espalda percepciones de ineficiencia, lentitud de respuesta y falta de capacidad para atender las demandas sociales.

De acuerdo con esta información, ¿cómo se ven afectados los trabajadores? En los estudios realizados por Fernández y Paravic (2003) y Mendoza (2015), se ha demostrado que la sobrecarga y el trabajo bajo presión, afectan la salud de los trabajadores en el ámbito físico como psicológico, lo cual produce un efecto en cadena, porque luego de verse afectado el trabajador, también se verá afectado el desarrollo óptimo de sus labores y con esto la calidad de la atención y sus relaciones interpersonales, facilitando el aumento de estrés laboral en los trabajadores. Por tanto, es necesario contar con sistemas que permitan reconocer lo que afecta al rendimiento de los empleados en el desarrollo de sus labores (Segredo, García, López, León y Perdomo, 2015). 
Este estudio busca explorar el efecto de los factores del clima organizacional en el estrés laboral, comparando funcionarios públicos del área de la salud y seguridad, debido a que las variables en estudio pueden afectar potencialmente el éxito de las labores de estos trabajadores. Tanto el área de salud como el de seguridad están expuestas a grandes niveles de exigencias, debido a que prestan un servicio que se relaciona directamente con las personas y en esa situación es importante el modo de la relación. Sin embargo, se pueden diferenciar estos dos sectores, principalmente por el nivel de formación académica requerida para ejercer en ellos y por el respeto social que adquieren por su labor.

\section{MARCO TEÓRICO}

\section{Clima organizacional}

Los elementos que influyen en un clima laboral no apropiado traen como consecuencia ausentismo, aumento en la rotación y disminución en el rendimiento de los empleados (Martínez, 2004). Por ello, la buena gestión del clima organizacional se ha convertido en una herramienta administrativa importante al momento de la toma de decisiones, permitiendo proyectar un aumento en la productividad, fortalecer la gestión de cambios en la organización y permitir un mejoramiento constante (Segredo y Pérez, 2007).

En tanto, diferentes investigadores que se han dedicado al estudio del clima organizacional han establecido su propia definición, objetivos, dimensiones, importancia, teorías e instrumentos para su medición, por consiguiente, cuando se estudia el clima organizacional se puede apreciar que no existe unificación teórica sobre su conceptualización (Segredo, 2013).

De acuerdo con Ramos (2012), los fundamentos teóricos básicos postulan que el comportamiento del individuo en el trabajo tiene que ver con la forma en que percibe el clima laboral y las características propias que este posea. Según Tagiuri (1968), el clima laboral es un aspecto del ambiente interno que se genera dentro de la organización, que experimentan sus miembros e influye en la conducta de estos. En ese sentido, se percibe el clima organizacional como un conjunto de propiedades del ambiente laboral, apreciado de forma directa o indirecta por los empleados. Respecto a ello, Chiavenato (2011) postula que el clima laboral se relaciona con la atmósfera generada en el ámbito interno de la organización.

Asimismo, Vega, Arévalo, Sandoval, Aguilar y Giraldo (2006) señalan que el clima organizacional es un fenómeno que agrupa las particularidades del ambiente físico que definen la personalidad de la institución y la distingue de otras. Por otro lado, Brunet (2011) señala el clima como un conjunto de características de una organización que son apreciadas por sus miembros, de acuerdo con la forma en la que la organización actúa con sus empleados.

Dentro de las diferentes conceptualizaciones de clima organizacional, Cuadra y Veloso (2007) señalan que es una percepción y es descriptiva. Por otro lado, Amaya y Martínez (2016), plantean que el clima organizacional surge de las interacciones entre los empleados y de estos con su entorno. De esta forma, para entender el clima de una institución de seguridad se deben considerar las características de la población y de la opinión pública. Martínez (2004), indica que el clima laboral está compuesto por varios elementos del trabajo relacionados con la satisfacción y que pueden ser una fuente de insatisfacción laboral como de estrés.

Para la elaboración de este estudio se trabajó sobre la base de la teoría de Koys y Decottis (1991), que señala que el clima describe la naturaleza de las percepciones que los empleados tienen de sus propias experiencias laborales. Como también indica que el clima es difícil de estudiar, debido a que es una variable compleja y multidimensional.

Este estudio trabaja con el clima agregado y considera percepciones individuales a nivel jerárquico en la organización.

\section{Estrés laboral}

El estrés es un factor que pertenece a los riesgos psicosociales y es el más ampliamente estudiado. Es un fenómeno relevante y reconocido socialmente, puesto que se ha convertido en uno de los principales problemas para la salud y la seguridad laboral, afectando la productividad y el deterioro de la calidad de vida en el personal sanitario que desarrolla su actividad profesional (Aguado, Bátiz y Quintana, 2013). De acuerdo con Sarafis y cols. (2016), el estrés afecta la relación entre satisfacción y placer que la persona tiene con su trabajo y con la calidad de vida. De igual forma, su percepción cambia según los momentos vividos, 
contemplando aspectos subjetivos e individuales (Vidotti, Trevisan, Quina, Perfeito y Cruz, 2019).

Para Peiró (1993), el estrés laboral es uno de los problemas de salud más grave, que no solo afecta a los trabajadores al provocarles incapacidad física o mental, sino también a los empleadores y los gobiernos, que comienzan a evaluar el perjuicio financiero que les causa. En ese sentido, la Organización Internacional del Trabajo (OIT, 2014) indica que esta enfermedad es uno de los problemas más importantes en muchos países y en diferentes tipos de lugares de trabajo, donde el estrés pone en riesgo la economía de la empresa y los países. Por tanto, el estrés tiene muchas consecuencias negativas porque afecta la salud física y mental de los trabajadores, lo que conlleva a una baja productividad de estos.

Por otra parte, la OMS (2013) define estrés laboral como la reacción que puede tener un individuo ante exigencias y presiones que no se ajustan a sus capacidades o conocimiento y ponen a prueba su habilidad para afrontar la situación. Bajo esa línea, se postula que el estrés laboral se produce por un desbalance sustancial entre la demanda y la capacidad de ejecución. Así, un efecto relevante del estrés en el área de salud es el agotamiento de los empleados, provocando la disminución de capacidades al momento de ofrecer calidad en la atención al paciente (Hall, Johnson, Watt, Tsipa y O'Connor, 2016).

López (2017) indica que el estrés en seguridad pública es un aspecto comúnmente relacionado con la eficacia de los funcionarios de seguridad, debido a que desempeñan labores importantes para la sociedad, y el interés por estudiar el estrés en seguridad pública se debe a que están expuestos a un ambiente de violencia lo cual implica mayores niveles de exigencia, carga laboral y emocional. Los cuales pueden producir estrés e insatisfacción laboral. Para Karasek (2001), el estrés es el resultado de la relación entre la alta demanda psicológica, el bajo poder de decisión (control) sobre el trabajo y el bajo apoyo social que recibe la persona de compañeros y jefaturas, es decir, existe un ambiente desagradable, tenso y con relaciones conflictivas.

De acuerdo con los diferentes estudios existentes, el clima organizacional y estrés laboral al que se ven involucrados los trabajadores del sistema público, pueden afectar directamente en la calidad de los servicios entregados (Aguado y cols., 2013; Martínez, 2004). Con base en lo anterior, el obje- tivo general de este estudio es explorar el efecto de los factores del clima organizacional en el estrés laboral, comparando funcionarios públicos del área de salud y seguridad. Para esto, se determina el nivel de estrés laboral en los trabajadores, se establece el grado de percepción con respecto al clima organizacional, y se entabla la relación existente entre las variables clima organizacional y estrés laboral. Como hipótesis se plantea que los factores del clima organizacional que afectan en el estrés laboral de los funcionarios del área de la salud son diferentes de los factores que afectan a los funcionarios de seguridad.

\section{METODOLOGÍA}

La encuesta fue aplicada en marzo de 2020, antes de la pandemia. La muestra está conformada por 321 individuos, correspondientes a funcionarios de salud y seguridad pública pertenecientes a la región del Biobío, de los cuales 136 son hombres $(42,4 \%)$ y 185 mujeres (57,6\%), con una edad promedio de 36,1 años y un rango entre 21 y 77 años. La antigüedad promedio en la institución es de 9.8 años con un rango entre 0,1 y 49 años de presencia. Se aplicaron dos escalas, la primera de clima organizacional y la segunda de estrés laboral, anteriormente aplicadas en Chiang y cols. (2007) y Chiang y cols. (2018), y fueron contestadas de manera voluntaria, anónima y sin control de tiempo. En la tabla 1 se muestran las características de los instrumentos de medición.

Para cumplir con el objetivo principal de este estudio, se aplicaron análisis de causalidad bajo el enfoque de Modelos de Ecuaciones Estructurales (SEM), que utilizan las covarianzas entre los ítems utilizados como dato de entrada, y efectúa inferencias respecto a los parámetros de causalidad definidos. Esta metodología es ampliamente usada para efectuar verificaciones empíricas potencialmente válidas para fenómenos en donde se midan constructos no tangibles (Hair y cols., 2010). En este tipo de aplicaciones se utilizan sistemas de ecuaciones considerando algunos criterios de optimalidad y de identificación, con el fin de que los resultados obtenidos sean coherentes a partir de la estructura teórica vinculada al fenómeno en estudio, en este caso, la vinculación del clima organizacional con el estrés laboral. Este enfoque permite relacionar variables en función de correlaciones estrictamente lineales dado que requiere de variables medibles y observables. 
Tabla 1: Características de los instrumentos de medición

\begin{tabular}{|c|c|c|c|c|}
\hline $\begin{array}{l}\text { Instrumento de } \\
\text { medición }\end{array}$ & Dimensiones & İtem & $\alpha$ & Tipo de respuesta \\
\hline \multirow{4}{*}{$\begin{array}{l}\text { Cuestionario de clima } \\
\text { organizacional } \\
\text { (Koys y Decottis, } 1991, \\
\text { adaptado y validado } \\
\text { por Chiang y cols., } \\
\text { 2008) }\end{array}$} & Apoyo (APO) & 2 & 0.845 & \multirow{4}{*}{$\begin{array}{l}\text { Escala Likert de } 1 \text { a } 5 \text {. Totalmente en } \\
\text { desacuerdo hasta muy de acuerdo }\end{array}$} \\
\hline & Innovación (INN) & 5 & 0.746 & \\
\hline & Confianza (CONF) & 3 & 0.721 & \\
\hline & Autonomía (AUT) & 2 & 0.791 & \\
\hline \multirow{3}{*}{$\begin{array}{c}\text { Cuestionario de Es- } \\
\text { trés Laboral } \\
\text { (Karasek, 1979) }\end{array}$} & $\begin{array}{c}\text { Apoyo Social Global Laboral } \\
\text { (ASGL) }\end{array}$ & 4 & 0.830 & \multirow{3}{*}{$\begin{array}{l}\text { Escala Likert de } 1 \text { a } 5 \text {. Totalmente en } \\
\text { desacuerdo hasta muy de acuerdo }\end{array}$} \\
\hline & Demanda Psicológica (DP) & 2 & 0.746 & \\
\hline & Control Laboral (CL) & 2 & 0.760 & \\
\hline
\end{tabular}

Fuente: Elaboración propia

La estimación de los modelos se divide en dos grupos: el primero para la muestra de funcionarios de salud, y el segundo para los funcionarios de seguridad pública. Para cada subgrupo existe un modelo, el cual permite comparar los coeficientes obtenidos a partir de un análisis de invarianza factorial. De esta forma, se estima la relación causal a través del método de máxima verosimilitud, con el propósito de determinar la magnitud y dirección de las relaciones entre variables, de cada variable respecto de su dimensión, y cada dimensión respecto a sus indicadores.

Los indicadores de bondad de ajuste utilizados fueron el índice chi-cuadrado, el índice chi-cuadrado corregido, RMSEA (Root Mean Square Error of Aproximation), SRMR (Standardized Root Mean Residual) y Comparative Fit Index. Luego, se efectuaron los análisis de invarianza, comparando los modelos obtenidos para cada grupo. Se ha establecido que habría diferencias estadísticamente significativas si $p<.05$. Luego, se calculan las medidas de fiabilidad y validez para cada modelo con el fin de comprobar estas propiedades psicométricas. Estos indicadores son Alfa de Cronbach e índice de fiabilidad compuesta para fiabilidad (deben ser $\geq 0.7$ ), y el índice de varianza extraída (debe ser $\geq 0.5$ ) para validez.
En tanto, la figura 1 muestra el modelo conceptual a estimar en función de las variables en estudio, y se aprecian cuatro variables exógenas (Apoyo, Innovación, Confianza y Autonomía), influyendo directamente sobre dos variables endógenas (Apoyo Social Clobal Laboral y Control Laboral), y finalmente una relación entre variables endógenas (Apoyo Social Global Laboral hacia Demanda Psicológica), totalizando ocho relaciones de causalidad directa. Se propone un efecto mediador de la confianza en dos relaciones: Innovación hacia Apoyo Social Global Laboral e Innovación hacia Control Laboral. También se propone un efecto mediador del Apoyo Social Global Laboral en las relaciones: Apoyo hacia Control Laboral y Apoyo hacia Demanda Psicológica, del mismo modo, se propone un efecto mediador del Apoyo Social Global Laboral en las relaciones: Innovación hacia Demanda Psicológica e Innovación hacia Control Laboral. 


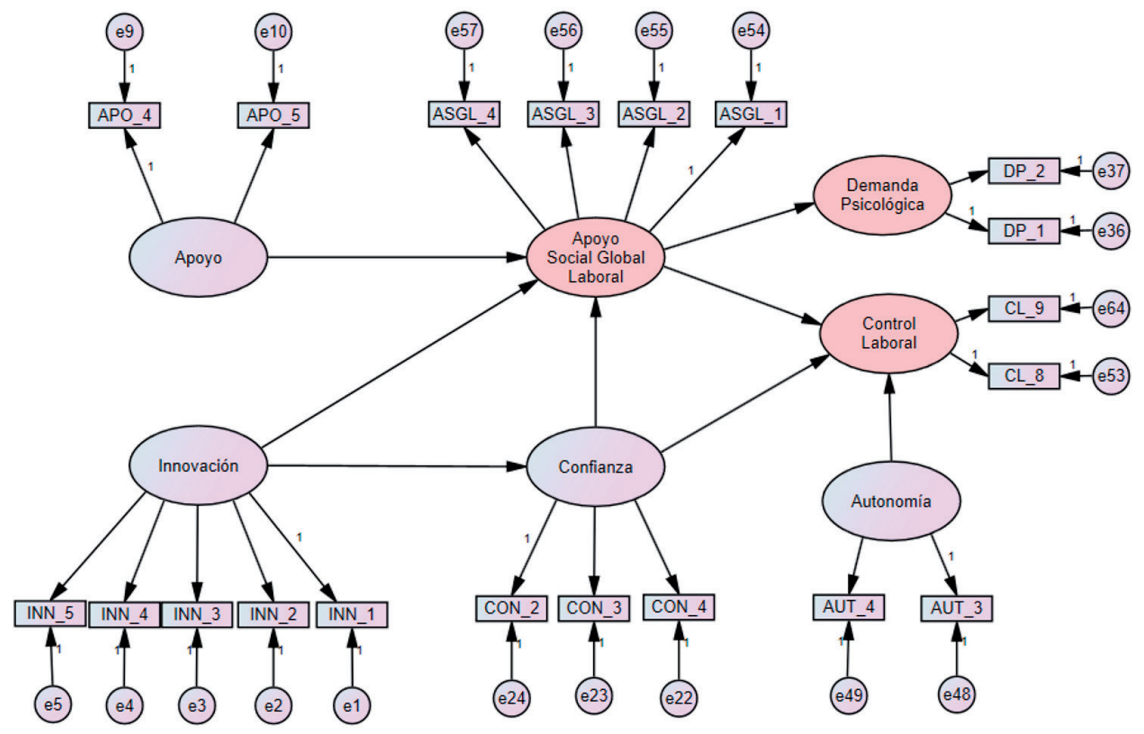

Figura 1: Modelo conceptual. Fuente: Elaboración propia con base en AMOS Software.

\section{RESULTADOS}

La estimación de los coeficientes definidos para las relaciones de causalidad sugiere los siguientes resultados:

\section{Bondad de ajuste}

La Tabla 2 representa el modelo en su conjunto y los resultados sugieren indicadores de ajuste aceptables (CFI=0.926; RMSEA=0.046), presentando entonces resultados relevantes para el análisis propuesto.

Tabla 2: Bondad de Ajuste

\begin{tabular}{ccc}
\hline Medida & Valores Ref. & Modelo \\
\hline$\chi 2$ & & $538.18(p=.000)$ \\
$\chi 2 / g . l$. & $2-5$ & 1.68 \\
CFI & $\geq 0.900$ & 0.926 \\
SRMR & $\leq 0.090$ & 0.078 \\
RMSEA & $\leq 0.080$ & 0.046 \\
\hline
\end{tabular}

Fuente: Elaboración propia.

\section{Cargas factoriales}

La Tabla 3 muestra un resumen de las cargas factoriales una vez validados los resultados obtenidos. De esta forma, los resultados sugieren que las cargas factoriales son todas significativas al $5 \%$, indicando su pertenencia a cada una de sus dimensiones. Por su parte, solo en los funcionarios de salud existe una representación importante de los ítems (carga superior a 0.6).
Dentro de cada variable, el clima organizacional tiene un buen comportamiento en los funcionarios de salud en cuanto a sus cargas factoriales superiores a 0.6 (100\% en todas), pero no en los funcionarios de seguridad, donde sus cargas factoriales solo alcanzan un $67 \%$ de magnitud superior a 0.6; para la variable estrés, para salud también tiene todos sus indicadores superiores a 0.6 y en Seguridad es de al menos un 75\%. 
Tabla 3: Resumen de Cargas Factoriales

\begin{tabular}{|c|c|c|c|c|c|c|}
\hline \multirow{2}{*}{ Variable } & \multirow{2}{*}{ Dimensión } & \multirow{2}{*}{ No Ítems } & \multicolumn{2}{|c|}{$\%$ Cargas $p<0.05$} & \multicolumn{2}{|c|}{$\%$ Cargas $>0.6$} \\
\hline & & & Salud & Seguridad & Salud & Seguridad \\
\hline \multirow{4}{*}{$\begin{array}{l}\text { Clima } \\
\text { Organiza- } \\
\text { cional }\end{array}$} & Apoyo (APO) & 2 & $100 \%$ & $100 \%$ & $100 \%$ & $0 \%$ \\
\hline & $\begin{array}{l}\text { Innovación } \\
\text { (INN) }\end{array}$ & 5 & $100 \%$ & $100 \%$ & $100 \%$ & $20 \%$ \\
\hline & $\begin{array}{l}\text { Confianza } \\
\text { (CON) }\end{array}$ & 3 & $100 \%$ & $100 \%$ & $100 \%$ & $67 \%$ \\
\hline & $\begin{array}{l}\text { Autonomía } \\
\text { (AUT) }\end{array}$ & 2 & $100 \%$ & $100 \%$ & $100 \%$ & $50 \%$ \\
\hline \multirow{3}{*}{$\begin{array}{l}\text { Estrés } \\
\text { Laboral }\end{array}$} & $\begin{array}{c}\text { Apoyo Social } \\
\text { Global Laboral } \\
\text { (ASGL) }\end{array}$ & 4 & $100 \%$ & $100 \%$ & $100 \%$ & $75 \%$ \\
\hline & $\begin{array}{c}\text { Demanda } \\
\text { Psicológica (DP) }\end{array}$ & 2 & $100 \%$ & $100 \%$ & $100 \%$ & $100 \%$ \\
\hline & $\begin{array}{c}\text { Control laboral } \\
(\mathrm{CL})\end{array}$ & 2 & $100 \%$ & $100 \%$ & $100 \%$ & $100 \%$ \\
\hline
\end{tabular}

Fuente: Elaboración propia.

\section{Fiabilidad y validez}

La Tabla 4 resume el análisis de fiabilidad y validez, los resultados sugieren que casi todos los indicadores de fiabilidad son superiores a 0.7 para los funcionarios de salud, no así para los de seguridad pública. Por su parte, los índices de varianza extraída son superiores en su mayoría a 0.5 , pero en el caso de los funcionarios de seguridad pública, solo dos superan el umbral. En ese sentido, los funcionarios de salud entregan información mucho más fiable y válida que los funcionarios de seguridad pública.

Tabla 4: Resumen de Fiabilidad y Validez

\begin{tabular}{cccccccc}
\hline \multirow{2}{*}{ Dimensión } & \multirow{2}{*}{$\mathrm{N}^{\circ}$ İtems } & \multicolumn{2}{c}{ Alfa de Cronbach } & \multicolumn{2}{c}{ Fiabilidad Compuesta } & \multicolumn{2}{c}{ Varianza Extraída } \\
\cline { 3 - 7 } & & Salud & Seguridad & Salud & Seguridad & Salud & Seguridad \\
\hline Apoyo & 2 & 0.787 & 0.377 & 0.835 & 0.394 & 0.786 & 0.145 \\
Innovación & 5 & 0.887 & 0.384 & 0.875 & 0.481 & 0.804 & 0.148 \\
Confianza & 3 & 0.814 & 0.686 & 0.814 & 0.725 & 0.841 & 0.129 \\
$\begin{array}{c}\text { Autonomía } \\
\text { Apoyo social }\end{array}$ & 2 & 0.712 & 0.495 & 0.704 & 0.502 & 0.554 & 0.283 \\
$\begin{array}{c}\text { global laboral } \\
\begin{array}{c}\text { Demanda } \\
\text { Psicológica }\end{array}\end{array}$ & 4 & 0.927 & 0.790 & 0.927 & 0.802 & 0.907 & 0.681 \\
$\begin{array}{c}\text { Control } \\
\text { Laboral }\end{array}$ & 2 & 0.797 & 0.687 & 0.802 & 0.687 & 0.733 & 0.535 \\
\hline
\end{tabular}

Fuente: Elaboración propia.

\section{Coeficientes del modelo}

Los coeficientes del modelo causal se muestran en la tabla 5. De acuerdo con los resultados obtenidos, para los funcionarios de salud existe un vínculo estadísticamente significativo de la in- novación sobre la confianza, la autonomía sobre el control laboral, y el apoyo social global laboral sobre la demanda psicológica. Para los funcionarios de seguridad pública, los resultados indican 
un efecto del apoyo sobre el apoyo social global laboral, la innovación sobre la confianza, la autonomía y el apoyo social global laboral sobre el clima laboral, y el apoyo social global laboral sobre la demanda psicológica.

Tabla 5: Coeficientes del Modelo

\begin{tabular}{|c|c|c|c|c|}
\hline \multirow{2}{*}{ Causalidad } & \multicolumn{2}{|c|}{ Seguridad Pública } & \multicolumn{2}{|c|}{ Salud } \\
\hline & Coef. & $\mathrm{R}^{2}$ & Coef. & $\mathrm{R}^{2}$ \\
\hline Apoyo Apoyo social Global Laboral & -0.295 & & $0.771^{*}$ & \\
\hline Innovación Apoyo social Global Laboral & 0.578 & $35.5 \%$ & -0.144 & $63.0 \%$ \\
\hline Confianza Apoyo social Global Laboral & 0.404 & & 0.241 & \\
\hline Innovación Confianza & $0.842^{* *}$ & $60.1 \%$ & $0.848^{* *}$ & $96.3 \%$ \\
\hline Confianza Control laboral & 0.095 & & -0.116 & \\
\hline Autonomía Control laboral & $0.585^{* *}$ & $26.5 \%$ & $0.454^{*}$ & $57.0 \%$ \\
\hline ASGL Control laboral & 0.145 & & $0.444^{*}$ & \\
\hline ASGL Demanda Psicológica & $-0.189^{*}$ & $1.4 \%$ & $-0.162^{*}$ & $3.4 \%$ \\
\hline
\end{tabular}

Fuente: Elaboración propia.

Específicamente, el control laboral es afectado por la autonomía y el apoyo social global laboral en los funcionarios de seguridad pública, mientras que para los de salud es solo la autonomía. Para ambos estamentos, un mayor apoyo social global laboral implica significativamente una menor demanda psicológica. Sin embargo, estos coeficientes a pesar de ser significativos no son lo suficientemente altos, indicando que existe mayor cantidad de factores que no han sido considerados.

El modelo ajustado de las variables en estudio se muestra en la figura 2.

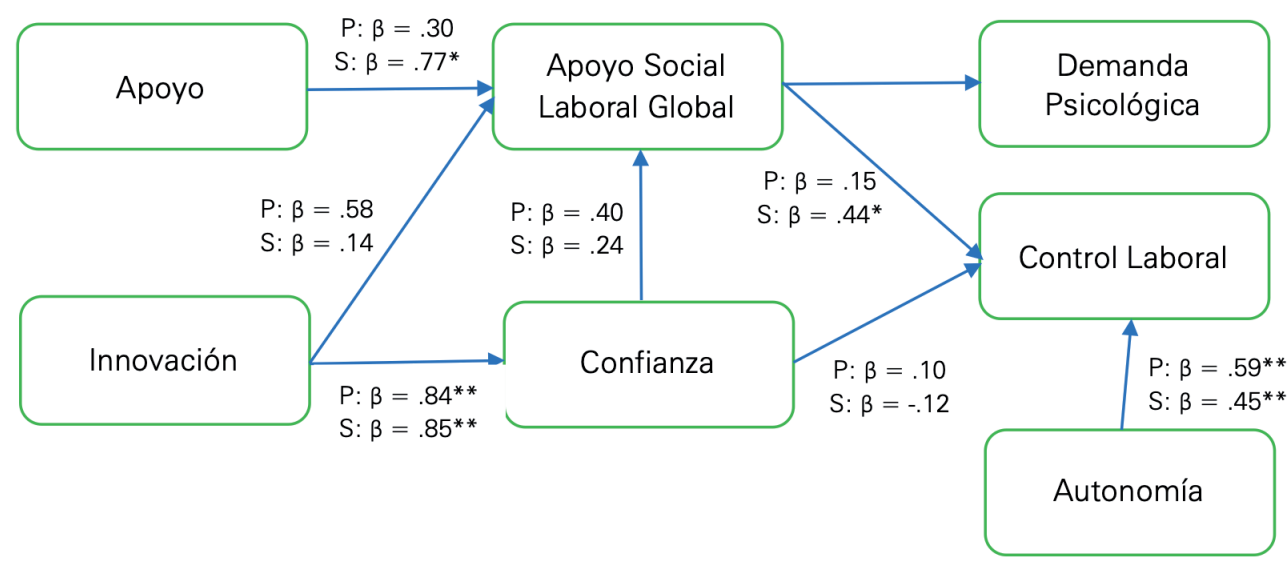

Figura 2: Modelo ajustado. Fuente: Elaborado a partir de AMOS Software.

\section{Análisis invarianza}

Finalmente, el análisis de invarianza permite comparar entre ambos tipos de funcionarios y evaluar si existen diferencias estadísticamente significativas entre las estructuras. La Tabla 6 muestra los resultados de las diferencias en los coeficientes causales $(p<$.001). En particular, en el caso de los funcionarios de salud, los coeficientes son significativamente distintos a los obtenidos para los funcionarios de seguridad pública. 
Tabla 6: Pruebas de invarianza

\begin{tabular}{cccc}
\hline Aspecto & $\chi 2$ & g. de I. & Valor $p$ \\
\hline Original & 538.18 & 100 & 0.000 \\
Coeficientes Causales Iguales & 582.24 & 79 & 0.000 \\
\hline
\end{tabular}

Fuente: Elaboración propia.

\section{CONCLUSIÓN Y DISCUSIÓN}

Se ha realizado un análisis estadístico de las relaciones entre variables de clima organizacional y de estrés, mediante el uso de modelación causal con ecuaciones estructurales. Los hallazgos indican que el apoyo social global laboral es un constructo que explica de forma positiva al control laboral en un $27 \%$ en el caso de la seguridad pública y un $57 \%$ para salud, y, además explica de forma negativa a la demanda psicológica en 1,4\% en la seguridad pública y un 3,4\% en salud.

Respecto de las propiedades psicométricas en las tres variables de estrés, estas se validan en todos sus ámbitos: fiabilidad, consistencia interna, validez, significación de los parámetros y bondad de ajuste. Sin embargo, para las variables de clima organizacional solo es posible confirmar la fiabilidad, pero no la validez de estas, indicando que los ítems de medición están vinculados a ella, pero no son los mejores.

Tras la estimación de los modelos por tipo de funcionario, la prueba de invarianza sugiere que existen diferencias estadísticamente significativas para los coeficientes causales. Por lo tanto, los funcionarios de la salud aportan más información a esta relación causal que los funcionarios de seguridad pública. Al respecto, el estrés laboral sí se ve afectado por el clima organizacional, pero en diferentes grados dependiendo de la dimensión y tipo de funcionario, por ejemplo, la dimensión apoyo afecta al estrés principalmente en el área de salud y la dimensión innovación afecta más a los funcionarios de seguridad pública.

En seguridad pública los factores de clima organizacional permiten explicar el apoyo social global laboral en un 35,5\%, donde los factores innovación y confianza son importantes para generar una explicación del apoyo laboral en el ámbito del estrés. En ese sentido, Amaya y Martínez (2016) destacan la importancia de la innovación, pues los individuos se desenvuelven bien en áreas como trabajo en equipo, supervisión y liderazgo, organi- zación y procesos de trabajo, relaciones entre dependencias, innovación y creatividad y relaciones interpersonales. De igual forma, Tang, Shao y Chen (2019) señalan que las empresas deben considerar medidas para promover y estimular el comportamiento innovador de los empleados, con el fin de mejorar efectivamente el conocimiento organizacional.

Para salud, las variables de clima organizacional permiten explicar el apoyo social global laboral en un $63 \%$, por lo tanto, los factores de clima organizacional: apoyo, innovación y confianza, son importantes para generar una explicación del apoyo laboral en el ámbito del estrés. Pedraza (2018) señala la importancia de factores apoyo y confianza, en particular, sugiere que los empleados públicos valoran el acompañamiento de los jefes hacia el empleado para recibir orientación, apoyo y confianza en el desarrollo de sus funciones. En ese sentido, se ha observado la importancia de líderes dentro de las organizaciones, porque estos influyen de forma positiva sobre las conductas de apoyo y de orientación a las reglas, y sobre la autonomía y profesionalismo, de tal forma que el clima organizacional mejore e influya en una mejor disposición de los trabajadores (Adriazola, López, Rojas y Bustamante, 2019).

Los resultados obtenidos en este estudio, junto a la evidencia existente, sugiere a las organizaciones considerar el clima organizacional que se presenta al interior de ella, como un ámbito importante para la gestión de los colaboradores, tanto para el área de la salud como el de seguridad. Cuando el clima organizacional no está alineado con los objetivos organizacionales puede afectar a las personas, desencadenando un bajo rendimiento laboral y afectar directamente el servicio que estas personas entregan a la sociedad. Para Zambrano, Ramón y Espinoza (2017), la importancia del clima dentro de la empresa ocupa un espacio relevante en la gestión de las organizaciones, por lo que es significativo estudiarlo 
para obtener un alto desempeño laboral, elevar el compromiso y la cooperación entre empleados.

Finalmente, Chiang y cols. (2017) plantean que el clima organizacional también tiene implicancias en la salud psicológica de los trabajadores, siendo los principales síntomas presentados el estrés y la fatiga. Se concluye que un clima organizacional adecuado beneficia la salud psicológica de los trabajadores.

Como limitación del presente estudio se puede destacar que es un estudio transversal, lo cual entrega una visión acotada. En ese sentido, se sugiere un estudio longitudinal que permita una visión de la tendencia existente. Quedan abiertas nuevas líneas de investigación como incluir más variables al estudio, satisfacción laboral, por ejemplo, o hacer una comparación entre salud pública y privada.

\section{REFERENCIAS}

Adriazola, F., López, M., Rojas, R. y Bustamante, M. (2019). Percepción del clima organizacional en atención primaria de salud en la región del Maule, Chile. Empresarial, 13(1), 13-21.

Aguado, J., Bátiz, A. y Quintana, S. (2013). El estrés en personal sanitario hospitalario; estado actual. Medicina y Seguridad del Trabajo, 59(231), 259-275.

Aguirre, G. (1990). Evaluación de la atención médica. Expectativas de los pacientes y de los trabajadores de la unidad médica. Salud Pública de México, 170-181.

Amaya, L. y Martínez, J. (2016). Una aproximación al clima organizacional en la Policía Nacional Civil (PNC) de El Salvador. Revista Policía y Seguridad Pública, 269-306.

Arnoletto, E. y Díaz, A. (2009). Un aporte a la gestión pública. Edición electrónica. http:// www.eumed.net/libros-gratis/2009b/550/ index.htm

Brunet, L. (2011). El Clima de Trabajo en las Organizaciones. México: Trillas.

Chiang, M., Heredia, S. y Santamaría, E. (2017) Clima organizacional y salud psicológica de los trabajadores: una dualidad organizacional. Dimensión empresarial, 15 (1),70-83.
Chiang, M., Riquelme, G. y Rivas, P. (2018). Relación entre Satisfacción Laboral, Estrés Laboral y sus Resultados en Trabajadores de una Institución de Beneficencia de la Provincia de Concepción. Ciencia \& Trabajo, 20(63), 178186.

Chiang, M., Salazar, C., y Núñez, A. (2007). Clima organizacional y satisfacción laboral en un establecimiento de salud estatal: hospital tipo 1. Theoria, 16(2), 61-76.

Chiang, M., Salazar, M., Huerta, P. y Núñez, A. (2008). Clima organizacional y satisfacción laboral en organizaciones del sector estatal (Instituciones Públicas). Desarrollo, adaptación y validación de instrumentos. Revista Universum de Universidad de Talca, 3(23), 6786.

Chiavenato, I. (2011). Administración de Recursos Humanos. México: McGraw Hill.

Cuadra, A. y Veloso, C. (2007). Liderazgo, clima y satisfacción laboral en las organizaciones. Universum: Revista de Humanidades y Ciencias Sociales, 2 (22), 40-56.

Fernández, B. y Paravic, T. (2003). Nivel de satisfacción laboral en enfermeras de hospitales públicos y privados de la provincia de Concepción, Chile. Ciencia y Enfermería, 9(2), 37- 48.

Ganga, F., Piñones, M., González, D. y Rebagliati, F. (2016). Rol del Estado frente al envejecimiento de la población: el caso de Chile. Convergencia, $23,175-200$.

Hall, L., Johnson, J., Watt, I., Tsipa, A. y O'Connor, D. (2016). Healthcare staff wellbeing, burnout, and patient safety: a systematic review. PLOS ONE, 11(7), 1-12.

Karasek, R. (1979). Job demand, job decision latitude and mental strain: implications for job redesign. Administrative Science Quarterly, 24(2), 285-308.

Karasek, R. (2001). El modelo de demandas/control: enfoque social, emocional y fisiológico del riesgo de estrés y desarrollo de comportamientos activos, en Centro Interamericano de Investigación y Documentación sobre Formación. Enciclopedia de Salud y Seguridad en el Trabajo, 2(34), 6-15.

Koys, D. y Decottis, T. (1991). Inductive Measures of Psychological Climate. Human Relations, 44(3), 265-385. 
López, A. (2017). Libros: El estrés y la toma de decisiones en los jefes y los líderes policiales. Revista Policía y Seguridad Pública, 495-499.

Malagón, G., Pontón, G. y Galán R. (2008). Administración Hospitalaria. Ed. medica panamericana.

Martínez, J. (2004). Estrés Laboral: Guía para empresarios y empleados. Madrid: Pearson Educación S.A.

Mendoza, R. (2015). Insatisfacción laboral como predictor del ausentismo en un hospital público. Revista Médica de Chile, 143, 1028-1033.

Montero, J. (2015). El concepto de seguridad en el nuevo paradigma de la normatividad mexicana. Región y Sociedad, 25(58), 203-238.

Organización Internacional del Trabajo (2014). La prevención del estrés en el trabajo: Lista de puntos de comprobación.

Organización Mundial de la Salud. (2013). Informe sobre la salud en el mundo 2013 - Investigaciones para una cobertura sanitaria universal.

Pedraza, N. (2018). El clima organizacional y su relación con la satisfacción laboral desde la percepción del capital humano. Revista Lasallista de Investigación, 15(1), 90-101.

Peiró, J. y Salvador, A. (1993). Control del estrés laboral. España: Editorial UDEMA S.A.

Ramos, D. (2012). El Clima organizacional: definición, teoría, dimensiones y modelos de abordaje. Reponame: Repositorio Institucional de La Universidad Nacional Abierta y a Distancia.

Rosas, M. (2012). ¿Cómo será (o debería ser) la agenda de seguridad del nuevo gobierno en México? En Atlas comparativo de la defensa en América Latina y el Caribe, coordinado por Marcela Donado. Buenos Aires: Resdal.

Sarafis, P., Rousaki, E., Tsounis, A., Malliarou, M., Lahana, L., Bamidis, P., Niakas, D. y Papastavrou, E. (2016). The impact of occupational stress on nurses' caring behaviors and their health-related quality of life. BMC Nursing, 15(1), 56.

Segredo, A. (2013). Clima organizacional en la gestión del cambio para el desarrollo de la organización. Revista Cubana de Salud Pública, 39(2), 385-393.
Segredo, A. y Pérez, L. (2007). El Clima organizacional en el desarrollo de los Sistemas Organizativos. INFODIR.

Segredo, A., García, A., López, P., León, P. y Perdomo, I. (2015). Enfoque sistémico del clima organizacional y su aplicación en salud pública. Revista Cubana de Salud Pública, 41 (1), 115-129.

Tagiuri, R. (1968). 'The concept of Organizational Climate'. En: R. Tagiuri y G. Litwin (Eds), Organizational Climate: Explorations of a Concept. Boston: Harvard University Division of Research, Graduate School of Business Administration.

Tang, Y., Shao, YF. y Chen YJ. (2019) Evaluar el mecanismo de mediación de la satisfacción laboral y el compromiso organizativo en el comportamiento innovador: la perspectiva del capital psicológico. Frontiers in Psychology, 10:2699, 1-12. .

Vega, D., Arévalo, A., Sandoval, J., Aguilar, M. y Giraldo, J. (2006). Panorama sobre los estudios de clima organizacional en Bogotá, Colombia. Revista Diversitas-Perspectivas en Psicología, 2, 329-349.

Vidotti, V., Trevisan, J., Quina, M., Perfeito, R., y Cruz, M. (2019). Síndrome de burnout, estrés laboral y calidad de vida en trabajadores de enfermería. Enfermería Global, 18(55), 344376.

Yáñez, J. (2003). Policía preventiva en la región central de México y el modelo gaditano de seguridad interior. University of California en San Diego: Center for U.S.-Mexican Studies.

Zambrano, J., Ramón, M. y Espinoza, E. (2017). Estudio sobre el clima organizacional en los docentes de la Universidad Técnica de Machala. Universidad y Sociedad, 9(2), 163-172. 
